\title{
Apollonius Tenth Problem as a Point Location Problem
}

\author{
Deok-Soo Kim ${ }^{1}$, Donguk Kim ${ }^{1}$, Kokichi Sugihara ${ }^{2}$, and Joonghyun Ryu ${ }^{1}$ \\ ${ }^{1}$ Department of Industrial Engineering, Hanyang University \\ 17 Haengdang-Dong, Sungdong-Ku, Seoul, 133-791 Korea \\ dskim@email.hanyang.ac.kr \\ \{donguk, jhryu\}@cadcam.hanyang.ac.kr \\ ${ }^{2}$ Department of Mathematical Engineering and Information Physics, Graduate School of \\ Engineering, University of Tokyo, 7-3-1, Hongo, Bunkyo-ku, Tokyo, 133 Japan \\ sugihara@simplex.t.u-tokyo.ac.jp
}

\begin{abstract}
Given a set of three circles in a plane, we want to find a circumcircle to these given circles called generators. This problem is well known as Apollonius Tenth Problem and is often encountered in geometric computations for CAD systems. This problem is also a core part of an algorithm to compute the Voronoi diagram of circles. We show that the problem can be reduced to a simple point-location problem among the regions bounded by two lines and two transformed circles. The transformed circles are produced from the generators via linear fractional transformations in a complex space. Then, some of the lines tangent to these transformed circles corresponds to the desired circumcircle to the generators. The presented algorithm is very simple yet fast. In addition, several degenerate cases are all incorporated into one single general framework.
\end{abstract}

\section{Introduction}

Suppose that we want to compute circumcircles of a set of three circles in a plane. The radii of the circles are assumed to be not necessarily equal and where the circles are possibly intersecting one another. This problem is frequently encountered in various geometric computations in CAD systems and the computation of the Voronoi diagram of circles $3,8,8,10,11,13,15,18]$.

The problem can be solved in various ways. One approach could be computing the center of circumcircle as an intersection between two bisectors defined by two circles. It turns out that this process involves the solution process of a quartic equation that can be solved by either the Ferrari formula or a numerical process [9]. Note that this approach can be applied only after the number of circumcircles to the generators is determined. On the other hand, the solution may be symbolically generated via tools like Mathematica. However, the cost of such symbolic generation can be also quite high.

It is known that there are at most eight circles simultaneously tangent to three circle generators as shown in Fig. 1. In this and the following figures, the black circles are given generator circles while the white ones are tangent circles.

Among the tangent circles, we want to find the circumcircles for three generator circles. Depending on the configuration of three generators, however, there may be 


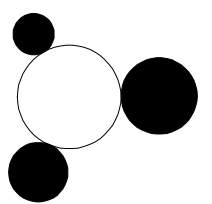

(a)

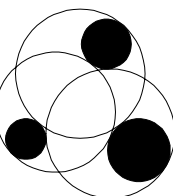

(b)

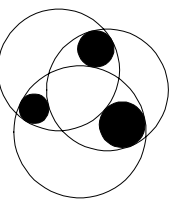

(c)

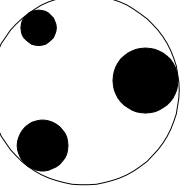

(d)

Fig. 1. Circles tangent to three generator circles

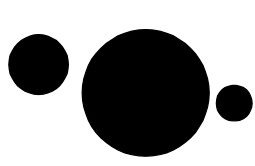

(a)

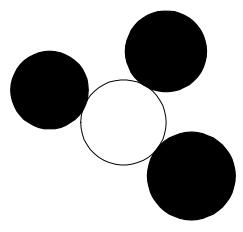

(b)

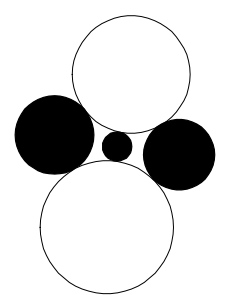

(c)

Fig. 2. Circumcircles. (a) no circumcircle exists, (b) one circumcircle exists, and (c) two circumcircles exist.

either no, one, or two circumcircles, as shown in Fig. 2. We want to determine which case a given generator set is and find such circumcircles with less computation as possible if they exist.

In Section 2, we provide the previous researches related to the problem. In Section 3 , the properties of the linear fractional transformation in a complex plane are provided so that the problem can be transformed to easier one. The discussions in this section is a slight variation of the noble approach initially presented by Rokne[16]. Based on the transformation, we present the point location problem formulation of the problem in Section 4.

\section{Related Works}

In his book On Contacts, Apollonius of Perga(262-190 B.C.), known as The Great Geometer, left the famous Apollonius problems : Given any three points, lines, or circles, or any combination of three of these, to construct a circle passing through the points and tangent to the given lines and circles. Among ten possible combinations of the geometric entities involved, The Apollonius' Tenth Problem is the most general problem to construct the circles simultaneously tangent to three circles. [2,4,5].

There have been several efforts to solve the problem in various ways $[1,3,14,17]$. Recently, Rokne reported an approach based on the linear fractional transformation (also known as Möbius transformation) in the complex plane [16]. Using the fact that a linear fractional transformation in a complex plane maps circles_o lines and vice versa, he suggested to compute a tangent line of two circles in a mapped space to back-transform into a circumcircle. Most recently, Gavrilova reported an analytic solution which involves trigonometric functions [7]. 
Even though the problem is quite complicated in Euclidean space, it turns out that it can be rather easily solved by employing a complex system. Following Rokne's suggestion, we have adopted the linear fractional transformation to transform the given problem into the problem of finding tangent lines of two circles in a mapped space. Then, we formulate a point location problem so that all of the degenerate configurations of generators can be handled in a unified way. It turns out that our approach incorporate all variations of degeneracies in a single framework, is easy to program, numerically robust, and computationally very efficient. Hence the proposed algorithm is preferable for the implementation for geometric computations.

\section{Linear Fractional Transformations}

Let the plane, where the circles are given, be complex. Then, a point $(x, y)$ in the Euclidean plane can be treated as a complex number $z=x+i y$. Also, let $\mathbf{c}_{i}=\left(z_{i}, r_{i}\right), i=$ 1,2 , and 3 , be the generator circles with a center $\left(x_{i}, y_{i}\right)$ and a radius $r_{1} \geq r_{2} \geq r_{3} \geq 0$ as shown in Fig. 3. Then, $\tilde{\mathbf{c}}_{i}=\left(z_{i}, r_{i}-r_{3}\right)$ transforms generator circles $\mathbf{c}_{1}, \mathbf{c}_{2}$ and $\mathbf{c}_{3}$ to shrunk circles $\tilde{\mathbf{c}}_{1}, \tilde{\mathbf{c}}_{2}$ and $\tilde{\mathbf{c}}_{3}$ respectively. Note that $\tilde{\mathbf{c}}_{3}$ degenerates to a point $z_{3}$. Then, if we can find a circle $\tilde{\mathbf{c}}$ passing through $z_{3} \equiv \tilde{\mathbf{c}}_{3}$ and tangent to both $\tilde{\mathbf{c}}_{1}$ and $\tilde{\mathbf{c}}_{2}$, we can easily find a circle $\mathbf{c}$ which is simultaneously tangent to $\mathbf{c}_{1}, \mathbf{c}_{2}$ and $\mathbf{c}_{3}$ by simply subtracting $r_{3}$ from the radius of $\tilde{\mathbf{c}}$.

Consider a linear fractional transformation defined as

$$
W(z)=\frac{a z+b}{c z+d}
$$

where $a d-b c \neq 0$, and $a, b, c$ and $d$ are either complex or real numbers. Note that $W(z)$ is analytic so that the mapping $W(z)$ is everywhere conformal and maps circles and straight lines in the $Z$-plane onto circles and straight lines in the $W$-plane. Among others, we note a particular linear mapping

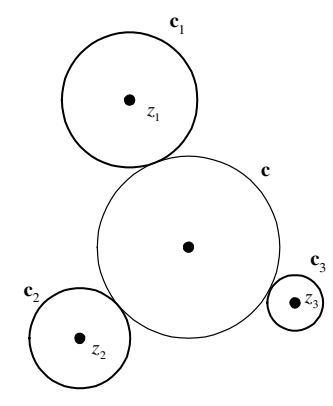

(a)

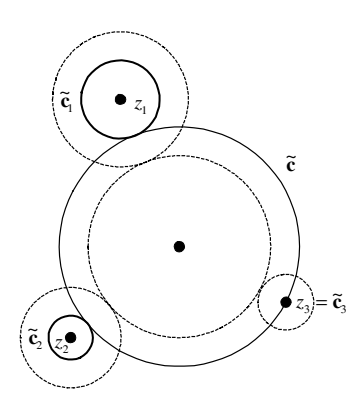

(b)

Fig. 3. Circumcircle and the inflated circumcircle. (a) generators and the desired circumcircle, (b) shrunk generators and a circumcircle passing through $z_{3}$. 


$$
W(z)=\frac{\square}{z-z_{0}}
$$

as was suggested by $[6,16]$. The mapping defined in Equation (2) is known to possess the following properties.

- It transforms lines and circles passing through $z_{0}$ in the Z-plane to straight lines in the $W$-plane.

- It transforms lines and circles not passing through $z_{0}$ in the $Z$-plane to circles in the $W$-plane.

- It transforms a point at infinity in the Z-plane to the origin of the $W$-plane.

The details can be found in a material on the subject such as [12]. Therefore, a mapping $W(z)=1 /\left(z-z_{3}\right)$ transforms $\tilde{\mathbf{c}}_{1}$ and $\tilde{\mathbf{c}}_{2}$ in the $Z$-plane to circles $W_{1}$ and $W_{2}$ in the $W$-plane, if $z_{3}$ is not on $\tilde{\mathbf{c}}_{1}$ and $\tilde{\mathbf{c}}_{2}$. Then, the desired circle $\tilde{\mathbf{c}}$ tangent to circles $\tilde{\mathbf{c}}_{1}$ and $\tilde{\mathbf{c}}_{2}$ in the $Z$-plane will be mapped to a line $L$ tangent to $W_{1}$ and $W_{2}$ in the $W$-plane by $W(z)$. It can be shown that $W(z)$ maps circles $\widetilde{\mathbf{c}}_{i}=\left(z_{i}, r_{i}-r_{3}\right)$ into circles $W_{i}=\left(\omega_{i}, R_{i}\right)$ defined as

$$
\begin{gathered}
\omega_{i}=\left(x_{i}-x_{3} / D_{i},-\left(y_{i}-y_{3}\right) / D_{i}\right) \\
R_{i}=\left(r_{i}-r_{3}\right) / D_{i}
\end{gathered}
$$

where $D_{i}=\left(x_{i}-x_{3}\right)^{2}+\left(y_{i}-y_{3}\right)^{2}-\left(r_{i}-r_{3}\right)^{2}, i=1$ and 2. Similarly, it can be also shown that the inverse transformation

$$
W^{-1}(z)=Z(w)=1 / w+z_{3}
$$

is also another conformal mapping, and hence, maps lines not passing through the origin of the $W$-plane to circles in the $Z$-plane. Suppose that a line is given as $a u+b v+1=0$ in the $W$-plane. Then, its inverse in the $Z$-plane is a circle $\tilde{\mathbf{c}}=\left(z_{0}, r_{0}\right)$, where $z_{0}=\left(-a / 2+x_{3}, b / 2+y_{3}\right)$ and $r_{0}=\sqrt{a^{2}+b^{2}} / 2$. We recommend [16] for the details of the computation using this mapping.

\section{Point Location Problem}

Based on Rokne's approach to transform Z-plane to $W$-plane, we formulate the problem as a point location problem. Let $W_{1}$ and $W_{2}$ be two circles with radii $R_{1}$ and $R_{2}$ in the $W$-plane, respectively. Suppose that $R_{1}>R_{2}>0$, as shown in Fig. 4(a). Then, there could be at most four distinct lines simultaneously tangent to both $W_{1}$ and $W_{2}$. Suppose that the black dot in Fig. 4(a) is the origin $O$ of the coordinate system in the $W$-plane. Then, the line $L_{1}$ maps to the circumcircle $\widetilde{\mathbf{c}}_{1}^{-1}$ in the $Z$-plane, as shown in Fig. 4(b), by the inverse mapping $Z(w)$ because the circles $W_{1}$ and $W_{2}$ as well as the origin $O$ are located in the same side with respect to $L_{1}$. Since the origin $O$ of the $W$ plane corresponds to infinity in the $Z$-plane and $Z(w)$ is conformal, $\tilde{\mathbf{c}}_{1}$ and $\tilde{\mathbf{c}}_{2}$ in the $Z$ plane are located to the infinity from the inverse mapped circle $\widetilde{\mathbf{c}}_{1}^{-1}$ and therefore $\widetilde{\mathbf{c}}_{1}^{-1}$ 


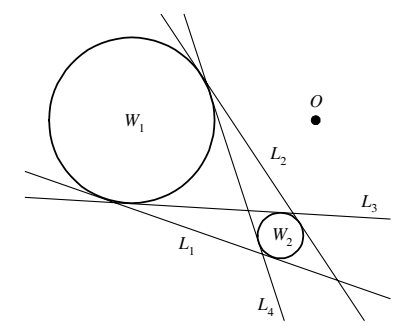

(a)

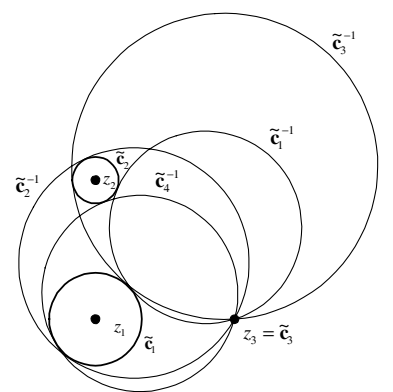

(b)

Fig. 4. $W^{-1}(z)=Z(w)=1 / w+z_{3}$ maps from the $W$-plane to the $Z$-plane. (a) the $W$-plane, (b) the $Z$-plane.

should be the desired circumcirlce. Therefore, we can also derive an observation of $O \notin\left(W_{1} \cup W_{2}\right)$, which means that the origin $O$ of the $W$-plane cannot lie on or interior to the circles $W_{1}$ and $W_{2}$.

Similarly, $L_{2}$ maps to the inscribing circle $\tilde{\mathbf{c}}_{2}^{-1}$ since the circles $W_{1}$ and $W_{2}$ are in the opposite side of $O$ which corresponds to the infinity in the Z-plane. Cases of $L_{3}$ and $L_{4}$ correspond to $\tilde{\mathbf{c}}_{3}^{-1}$ and $\widetilde{\mathbf{c}}_{4}^{-1}$, respectively. Therefore, the line $L$ which corresponds to a circumcircle in the Z-plane is either one or both of the exterior tangent lines, $L_{1}$ and/or $L_{2}$. Between $L_{1}$ and $L_{2}$, the one containing $W_{1}, W_{2}$ and the origin $O$ in the same side of the line will map to the desired circumcircle(s). Remember that zero, one or both exterior tangent lines may be the correct result depending on the configuration of the initially given generator circles. From now on, we will drop the word exterior from the term for the convenience of presentation, unless otherwise needed.

\subsection{Decomposition of the $W$-Plane}

Suppose $W_{1}$ and $W_{2}, R_{1}>R_{2} \neq 0$, are given as shown in Fig. 5(a). Let $L_{1}$ and $L_{2}$ be the tangent lines to both circles. Let $L_{i}^{+}$be the half-space, defined by $L_{i}$, containing $W_{1}$ as well as $W_{2}$. Similarly, $L_{i}^{-}$means the opposite side of $L_{i}^{+}$. Then, $W$-plane consists of six mutually exclusive regions as follows:

$$
\begin{array}{lll}
\alpha=\left(L_{1}^{+} \cap L_{2}^{-}\right) \cup\left(L_{1}^{-} \cap L_{2}^{+}\right) & \beta=L_{1}^{-} \cap L_{2}^{-} & \gamma=L_{1}^{+} \cap L_{2}^{+} \\
\delta=L_{1} \cap L_{2} & \varepsilon=\left(L_{1} \cap L_{2}^{-}\right) \cup\left(L_{1}^{-} \cap L_{2}\right) & \zeta=\left(L_{1} \cap L_{2}^{+}\right) \cup\left(L_{1}^{+} \cap L_{2}\right)
\end{array}
$$

As shown in the figure, the region $\alpha$ consists of two subregions and the region $\gamma$ consists of three (or four, if $W_{1}$ and $W_{2}$ intersect each other) subregions.

\subsection{Location of the Origin of the W-Plane}

Once the $W$-plane is decomposed into a set of such regions, the problem of computing a circumcircle(s) now further reduces to a point location problem among the regions. 
Note that, in Fig.5, the shaded circles are shrunk circles, and black dots are the shrunk circles with zero radii and thus degenerate to a point in the Z-plane. In addition, a circumcircle is shown in a solid curve while an inscribing circle is shown in a broken curve.

Theorem 1. If $R_{1}>R_{2} \neq 0$, there are six cases as follows.

- Case $\alpha$ : If $O \in \alpha$, one tangent line maps to a circumcircle while the other tangent line maps to an inscribing circle. (Fig. 5(b)- $\alpha$ )

- Case $\beta$ : If $O \in \beta$, both tangent lines map to inscribing circles. (Fig. 5(b)- $\beta$ )

- Case $\gamma$ : If $O \in \gamma$, both tangent lines map to circumcircles. (Fig. 5(b)- $\gamma$ )

- Case $\delta$ : If $O \equiv \delta$, both tangent lines map to lines intersecting at a point. (Fig. 5(b)$\delta)$

- Case $\varepsilon$ : If $O \in \mathcal{E}$, a tangent line on which $O$ lies maps to a line, while the other tangent line maps to an inscribing circle. (Fig. 5(b)- $\varepsilon$ )

- Case $\zeta$ : If $O \in \zeta$, the tangent line on which $O$ lies maps to a line, while the other tangent line maps to a circumcircle. (Fig. 5(b)- $\zeta$ )

\section{Proof.}

- Case $\alpha$ : Suppose that $\alpha_{1}=\left(L_{1}^{-} \cap L_{2}^{+}\right)$and $\alpha_{2}=\left(L_{1}^{+} \cap L_{2}^{-}\right)$. Without loss of generality we can assume that $O \in \alpha_{1}$. Then, $L_{1}$ in the $W$-plane is inverse-mapped to a circle $\tilde{\mathbf{c}}_{1}^{-1}$ inscribing $\tilde{\mathbf{c}}_{1}$ and $\tilde{\mathbf{c}}_{2}$ in the Z-plane, as illustrated by a dotted curve in Fig.5(b)- $\alpha$. This is because $L_{1}$ places $O$ on the opposite side of $W_{1}$ and $W_{2}$. Note that $\tilde{\mathbf{c}}_{1}$ and $\tilde{\mathbf{c}}_{2}$ are the inverse maps of $W_{1}$ and $W_{2}$. On the other hand, $L_{2}$ is inverse-mapped to a circumcircle $\tilde{\mathbf{c}}_{2}^{-1}$ tangent to $\widetilde{\mathbf{c}}_{1}$ and $\widetilde{\mathbf{c}}_{2}$ in the $Z$-plane, and is illustrated as a solid curve. This is because $L_{2}$ places $W_{1}, W_{2}$ and $O$ on the same side. Since two tangent lines in the $W$-plane intersect each other at $\delta$, the inverse mapped circles, regardless they are circumcircles or inscribing circles, always intersect each other at $W^{-1}(\delta)$ computed by Eq.(4) shown as a black rectangle in the Z-plane.

- Case $\beta$ : When $O \in \beta$, both $W_{1}$ and $W_{2}$ are on the opposite side of $O$ with respect to both tangent lines $L_{1}$ and $L_{2}$. Therefore, both $L_{1}$ and $L_{2}$ should be mapped to inscribing circles, and hence, no circumcircle will result as shown in Fig. 5(b)- $\beta$.

- Case $\gamma$ : When $O \in \gamma$, both $W_{1}$ and $W_{2}$ are on the same side of $O$ with respect to both tangent lines $L_{1}$ and $L_{2}$. Hence, both $L_{1}$ and $L_{2}$ should be mapped to circumcircles only. In this case, two different situations may occur. Note that the region $\gamma$ consists of three subreigons. The case in Fig. 5(b)- $\gamma_{1}$ occurs when $O$ lies in-between two circles $W_{1}$ and $W_{2}$, and the case $\gamma_{2}$ in Fig. 5(b)- $\gamma_{2}$ occurs when $O$ lies in the other subregions of $\gamma$.

- Case $\delta$ : When $O \equiv \delta$, the inverse mapping to the $Z$-plane yields results similar to what is shown in the $W$-plane. Since the tangent lines in $W$-plane pass through the origin $O$, the inverse-mapped (supposedly) circles should pass through the infinity. This means that the radii of the inverse-mapped circles are infinite. Therefore, the mapping results in lines in Z-plane as shown in Fig. 5(b)- $\delta$. Note that they only intersect at $\widetilde{\mathbf{c}}_{3}$. 


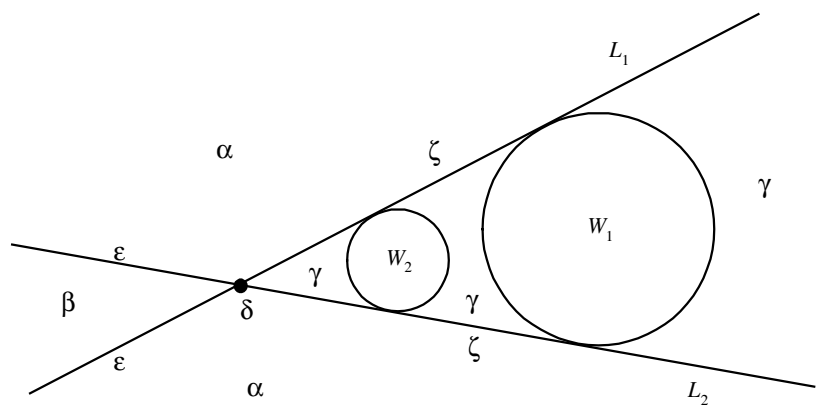

(a)

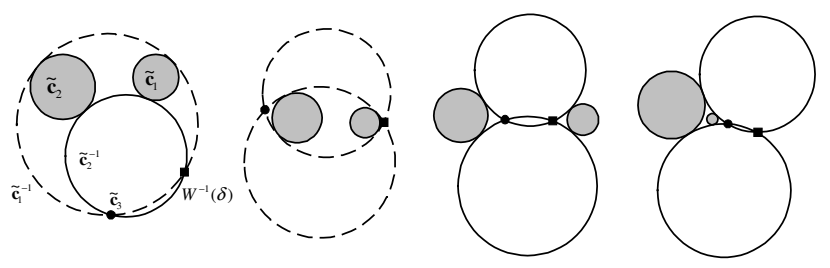

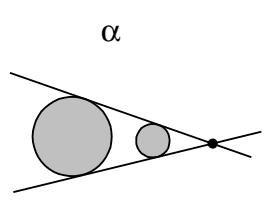

$\delta$

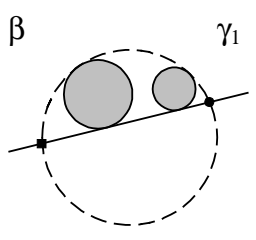

$\varepsilon$

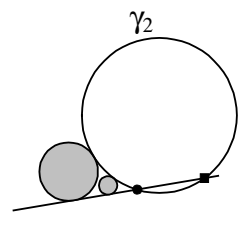

$\zeta$

(b)

Fig. 5. $R_{1}=R_{2}>0$. (a) the $W$-plane, (b) the $Z$-plane

- Case $\varepsilon$ : When $O \in \varepsilon, O$ lies precisely on a ray $\varepsilon$ starting from $\delta$. In this case, the corresponding tangent line on which $O$ lies is inverse mapped to a line in the $Z$ plane, as was explained in the above. Then, $O$ should be located on the opposite side of the other tangent line with respect to $W_{1}$ and $W_{2}$, meaning that there is an inscribing circle as shown in Fig. 5(b)- $\varepsilon$.

- Case $\zeta$ : When $O \in \zeta, O$ lies precisely on a ray $\zeta$, which is also a ray starting from $\delta$. In this case, the corresponding tangent line inverse maps to another line in the $Z$ plane similarly to the above cases. In this case, however, $O$ as well as $W_{1}$ and $W_{2}$ should be located on the same side of the other tangent line. It means that the tangent line inverse maps to a circumcircle in the Z-plane as shown in Fig. 5(b)- $\zeta$.

Note that some tangent circles to shrunk circles degenerate to lines in Cases $\delta$, $\varepsilon$ and $\zeta$. In this case, the desired tangent circles to the generators can be obtained by translating the degenerate lines to the opposite direction of the shrunk circles.

Slightly changing the configuration of generator circles, various degeneracies may occur. It turns out that the degeneracies are mainly due to the radii of $W_{1}$ and $W_{2}$. 


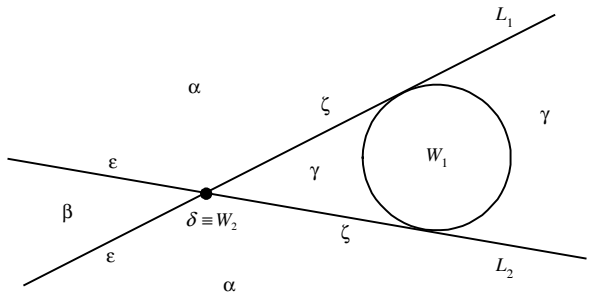

Fig. 6. $R_{1}>R_{2}=0$.

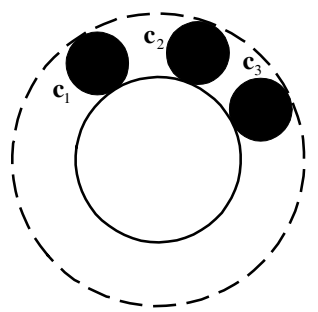

(a)

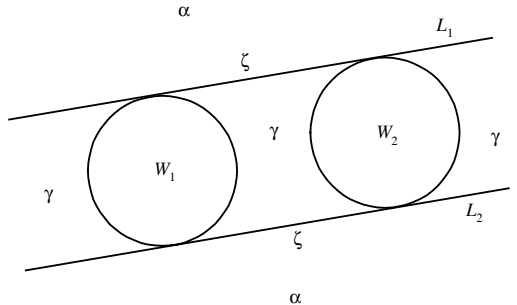

Fig. 7. $R_{1}=R_{2}>0$

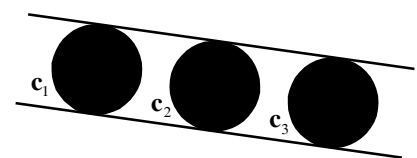

(b)

Fig. 8. $R_{1}=R_{\imath}=0:$ generator circles in the $Z$-plane

\subsection{Degenerate Cases}

Even though the problem has been discussed for a general case, there could be several degeneracies which may make the problem more difficult. The degeneracies are mainly due to the radii of $W_{1}$ and $W_{2}$. It turns out that, however, the theory previously discussed can be used for such degeneracies without much modifications.

One degenerate case is $R_{1}>R_{2}=0$, which means that $W_{2}$ degenerates to a point as shown in Fig. 6. This case occurs when two smaller generator circles $\mathbf{c}_{2}$ and $\mathbf{c}_{3}$ in the $Z$-plane have identical radii. The differences of this case from the general case are the followings: i) The region $\gamma$ consists of two subregions, and ii) Case $\delta$ does not occur. Otherwise, everything is same as before.

A second degenerate case occurs when $R_{1}=R_{2}>0$, as shown in Fig. 7, which means that $W_{1}$ and $W_{2}$ have identical non-zero radii. Note that $R_{1}=R_{2}$ in general does not guarantee $r_{1}=r_{2}$, which are the radii of generator circles. In other words, even though two generator circles in the $Z$-plane have identical radii, the radii of mapped circles in the $W$-plane are not necessarily identical, and vice versa. Note that two exterior tangent lines in the $W$-plane are parallel in this case. In this case, therefore, the regions $\beta, \delta$, and $\varepsilon$ disappear. Therefore, the cases left are Cases $\alpha, \gamma$, and $\zeta$, and Theorem 1 still holds except the missing cases.

A third, and the last, degenerate case is $R_{1}=R_{2}=0$, and is illustrated in Fig. 8. This case occurs when both $W_{1}$ and $W_{2}$ have zero radii, and therefore $L_{1} \equiv L_{2}$. This case is possible only when all generator circles in the Z-plane have identical radii. In this case, only the regions $\alpha$ and $\zeta$ only remain. The interpretations of the remaining 
regions stay the same as before. Note that Fig. 6 and Fig. 7 illustrate the $W$-plane while Fig. 8 shows the $Z$-plane.

Therefore, these degenerate cases can be all treated in a unified algorithm without any modification, except the minor treatment of parsing the regions. One possible special treatment would be the very last case where the centers of three circles with identical radii are collinear. In this case, there are no circumcircle but two tangent lines, as shown in Fig. 8.(b), and they can be only computed by translation of the computed line.

\section{Conclusions}

Presented in this paper is an algorithm to compute the circumcircles of a set of three generator circles in a plane. This problem is a part of the well-known Apollonius' Tenth Problem and is frequently encountered in various geometric computations for CAD systems as well as for the computation of the Voronoi diagram of circles. It turns out that this seemingly trivial problem is not an easy problem at all to solve in a general setting. In addition, there can be several degenerate configurations of the generators.

Even though the problem is quite complicated in Euclidean space, it turns out that it can be rather easily solved by employing a complex system. Following Rokne's approach, we have adopted the linear fractional transformation to transform the given problem into the problem of finding tangent lines of two circles in a mapped space. Then, we formulate a point location problem so that all of the degenerate configurations of generators can be handled in a unified way.

It turns out that the proposed approach incorporates all variations of degeneracies in a single framework, is easy to program, numerically robust, and computationally very efficient. We have also demonstrated the validity and efficiency of the algorithm by applying the theory to the computation of Voronoi diagram of circles.

We expect that the idea presented in this paper can extend to all Apollonius Problems to solve them in a single general framework, as far as the circumcircle is concerned.

\section{Acknowledgements}

The first author was supported by Korea Science and Engineering Foundation (KOSEF) through the Ceramic Processing Research Center (CPRC) at Hanyang University, and the third author was supported by Torey Science Foundation, Japan.

\section{References}

1. Altshiller-Court, N., The problem of Apollonius. College Geometry, $2^{\text {nd }}$ Ed., Barnes and Noble, New York, (1952) 173-181. 
2. Boyer, C. B., A History of Mathematics, Wiley, New York (1968).

3. Capelli, R. Circle tangential to 3 cicles or lines. Posting No. 35067, Usenet newsgroup comp.graphics.algorithms, 2 pages, (1996).

4. Courant, R. and Robbins, H., What is Mathematics?: An Elementary Approach to Ideas and Methods, $2^{\text {nd }}$ edition, Oxford University Press, Oxford (1996).

5. Dörrie, H., 100 Great Problems of Elementary Mathematics: Their History and Solutions, Dover, New York (1965).

6. Gavrilova, M. and Rokne, J., Swap conditions for dynamic Voronoi diagram for circles and line segments, Computer Aided Geometric Design, Vol. 16 (1999) 89-106.

7. Gavrilova, M. and Rokne, J., Apollonius' Tenth Problem Revisited, Special Session on Recent Progress in Elementary Geometry, 941 ${ }^{\text {st }}$ American Mathematical Society Conference (1999) 64

8. Kim, D.-S., Hwang, I.-K. and Park, B.-J., Representing the Voronoi diagram of a simple polygon using rational quadratic Bézier curves, Computer-Aided Design, Vol. 27, No. 8 (1995) 605-614.

9. Kim, D.-S., Lee, S.-W. and Shin, H., A cocktail algorithm for planar Bézier curve intersections, Computer-Aided Design, Vol. 30, No. 13 (1998) 1047-1051.

10. Kim, D.-S., Kim, D. and Sugihara, K., Voronoi diagram of a circle set from Voronoi diagram of a point set: I. Topology, (Submitted to Computer Aided Geometric Design 2001).

11. Kim, D.-S., Kim, D. and Sugihara, K., Voronoi diagram of a circle set from Voronoi diagram of a point set: II. Geometry, (Submitted to Computer Aided Geometric Design 2001).

12. Kreyszig, E., Advanced Engineering Mathematics, $7^{\text {th }}$ Edition, John Wiley \& Sons (1993).

13. Lee, D.T. and Drysdale, R.L., III, Generalization of Voronoi diagrams in the plane, SIAM J. COMPUT., Vol. 10, No. 1 (1981) 73-87.

14. Moise, E.E., Elementary Geometry from an Advanced Standpoint, $3^{\text {rd }}$. ed., AddisonWesley Publ. Co., Reading (1990).

15. Okabe, A., Boots, B. and Sugihara, K., Spatial Tessellations Concepts and Applications of Voronoi Diagram, John Wiley \& Sons (1992).

16. Rokne, J., Appolonius's 10th problem, Graphics Gems II, ed. James Arvo, Academic Press, (1991) 19-24.

17. Sevici, C.A., Solving the problem of Apollonius and other related problems, Graphics Gems III, ed. David Kirk, Academic Press, San Diego (1992) 203-209.

18. Sharir, M., Intersection and closest-pair problems for a set of planar discs, SIAM J. COMPUT., Vol. 14, No. 2 (1985) 448-468. 